\title{
Monitored transoral endoscopic thyroidectomy via long monopolar stimulation probe
}

\author{
Gianlorenzo Dionigi ${ }^{1}$, Che-Wei Wu ${ }^{2,3}$, Ralph P. Tufano ${ }^{3}$, Antonio Giacomo Rizzo ${ }^{1}$, Angkoon Anuwong ${ }^{4}$, \\ Hui Sun ${ }^{5}$, Paolo Carcoforo ${ }^{6,7}$, Cancellieri Antonino ${ }^{1}$, Mattia Portinari ${ }^{6,7}$, Hoon Yub Kim ${ }^{8}$ \\ ${ }^{1}$ Division for Endocrine and Minimally Invasive Surgery, Department of Human Pathology in Adulthood and Childhood "G. Barresi", University \\ Hospital G. Martino, University of Messina, Messina, Italy; ${ }^{2}$ Faculty of Medicine, College of Medicine, Kaohsiung Medical University Hospital, \\ Kaohsiung Medical University, Kaohsiung, Taiwan; ${ }^{3}$ Department of Otolaryngology-Head and Neck Surgery, Kaohsiung Medical University \\ Hospital, Kaohsiung Medical University, Kaohsiung, Taiwan; ${ }^{4}$ Division of Head and Neck Endocrine Surgery, Department of Otolaryngology- \\ Head and Neck Surgery, Johns Hopkins University School of Medicine, Baltimore, Maryland, USA; ${ }^{5}$ Minimally Invasive and Endocrine Division, \\ Department of Surgery, Police General Hospital, Bangkok, Thailand; ' Division of Thyroid Surgery, Jilin Provincial Key Laboratory of Surgical \\ Translational Medicine, China Japan Union Hospital of Jilin University, Changchun 130033, China; ${ }^{7}$ Department of Surgery, S. Anna University \\ Hospital, Ferrara, Italy; ${ }^{8}$ Department of Morphology, Surgery, and Experimental Medicine, University of Ferrara, Ferrara, Italy; ${ }^{9}$ Department \\ of Surgery, Division of Breast and Endocrine Surgery, Minimally Invasive Surgery and Robotic Surgery Center, KUMC Thyroid Center Korea \\ University, Anam Hospital, Seoul, Korea \\ Correspondence to: Prof. Gianlorenzo Dionigi, MD, FACS. Division for Endocrine and Minimally Invasive Surgery, Department of Human Pathology \\ in Adulthood and Childhood "G. Barresi", University Hospital G. Martino, University of Messina, Via C. Valeria 1, 98125, Messina, Italy. \\ Email: gdionigi@unime.it.
}

\begin{abstract}
This video aimed to describe the role of intraoperative neuromonitoring (IONM) during transoral endoscopic thyroidectomy vestibular approach (TOETVA) with emphasis given to IONM technical and technological notes, the identification of recurrent laryngeal nerve (RLN). Standardized technique of IONM consist in identifying and monitoring both the vagus nerve and the RLNs before and after resection (V1, V2, R1, R2). According to this report, IONM during TOETVA is feasible and safe in providing identification and function of laryngeal nerves. IONM enable surgeons to feel more comfortable with their initial approach to TOETVA or extended indications. Larger series are needed for appropriated evaluation of IONM in reduction of the rates for RLN complications.
\end{abstract}

Keywords: Thyroid; thyroidectomy; mini-invasive thyroidectomy; transoral thyroidectomy; intraoperative neural monitoring; standardization

Received: 26 October 2017; Accepted: 27 December 2017; Published: 26 January 2018.

doi: 10.21037 /jovs.2017.12.25

View this article at: http://dx.doi.org/10.21037/jovs.2017.12.25

\section{Introduction}

The purpose of this visualized paper is primarily to describe technical tips, advices and the value of using intraoperative nerve monitoring (IONM) during transoral endoscopic thyroidectomy vestibular approach (TOETVA), with emphasis and respect given to nerve monitoring standards described by the International Neural Monitoring Study Group (INMSG) (1-23).

\section{Patient selection and workup}

Indications for TOETVA are as follows: a predicted gland width on diagnostic imaging $\leq 10 \mathrm{~cm}$; a thyroid volume outline of $<45 \mathrm{~mL}$ or dominant nodule dimension of $\leq 50 \mathrm{~mm}$; Bethesda category 3 or 4 lesions; primary papillary microcarcinoma without local or distant metastasis; patient request for optimal aesthetic results (1-16). Exclusion criteria are as follows: patients unfit for 

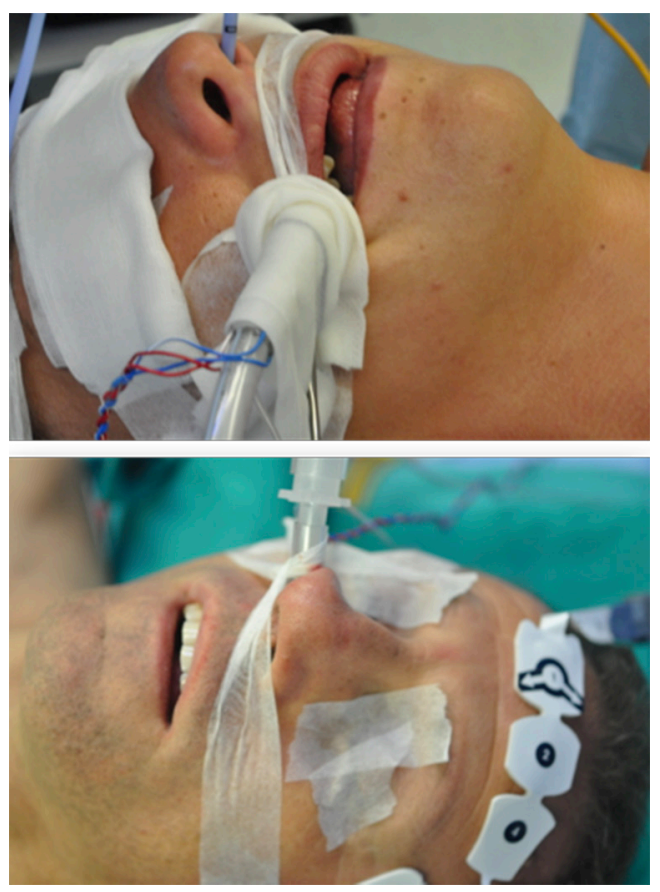

Figure 1 Naso- or orotracheal intubation with the EMG endotracheal tube. EMG, electromyography.

general anesthesia; precedent radiation in the head, neck, or upper mediastinum; antecedent neck surgery; recurrent goiter; a gland volume of $>45 \mathrm{~mL}$ or main nodule diameter of $>50 \mathrm{~mm}$; documentation of lymph node or distant metastases, tracheal/esophageal infiltration, preoperative laryngeal nerve palsy, hyperthyroidism, mediastinal goiter, or oral abscesses. Moreover, patients with poorly- or undifferentiated cancer, dorsal extrathyroidal radius, and/or lateral neck metastasis (N1b) are not favored for TOETVA (1-16).

Candidates for TOETVA undergo same preoperative workup will offer a conventional open approach.

\section{Preoperative preparation}

The 3 vestibular incisions determine a new communication between the oral cavity and neck and a poor oral heath may contribute the ability of the oral microbiota to invade the body. Good oral and dental hygiene can prevent possible postoperative infective complications. Therefore, it is suggested a preoperative oral/dental assessment by specialist. Patients undergo dental care about 1 month before TOETVA (1-9). This is recommended especially in high risk subjects as diabetic and cardiovascular patients.
Moreover, pre- and post-operatively, patients are asked to gargle with Chlorhexidine mouth wash. Amoxicillin/ clavulanic acid is used for preoperative prophylactic antibiotic (1-9). Some authors suggest extending the antibiotic for 5 days postoperatively orally (1-9).

\section{Equipment preference card}

Monitored TOETVA is performed according to standards of equipment set up, induction and maintenance anesthesia, correct tube positioning verification tests, EMG definitions described by the INMSG Guidelines (23).

Nerve-monitoring formats as laryngeal palpation, glottic observation, glottic pressure monitoring, endoscopically placed intramuscular vocal cord electrodes, intramuscular electrodes placed through the cricothyroid membrane, and postcricoid surface electrodes are difficult to use in any endoscopic thyroid procedure $(21,23,24)$. Thus, for safety, utility, simplicity, systems that rely on endotracheal tube-based surface electrodes were preferred monitoring equipment format for TOETVA.

Pre-fashioned with integrated paired left and right stainless steel electrodes embedded within the endotracheal tube surface were used (NIM TriVantage ${ }^{\circledR}$ EMG Tube, Medtronic, Jacksonville, Florida, USA). The electrodes are connected to the IONM station (NIM3.0, Medtronic, Jacksonville, Florida, USA).

\section{Procedure, tips, trics and pitfalls}

TOETVA is performed under general anesthesia with naso- or orotracheal intubation (Figure 1). The EMG endotracheal tube is fixed at the right corner of the mouth and equipment for anesthesia is set up on the same side of the patient. The endotracheal tube tape is fixed on the upper lip, not on the lower as the last is involved during dissection.

The operative room setting included the IONM monitor at patients' foot (Figure 2).

A single use, $230 \mathrm{~mm}$ ball tip long stimulation probe, monopolar (product n.PSP1002, Medtronic, Jacksonville, Florida, USA) was used in this video for nerve stimulating (Figures 3,4). The probe is connected with the IONM system.

For the right handed surgeon, the long stimulating probe is introduced and used from the right port without any interference with the other endoscopic instruments (Figure 5).

The advantages of using the long stimulating probe are: 


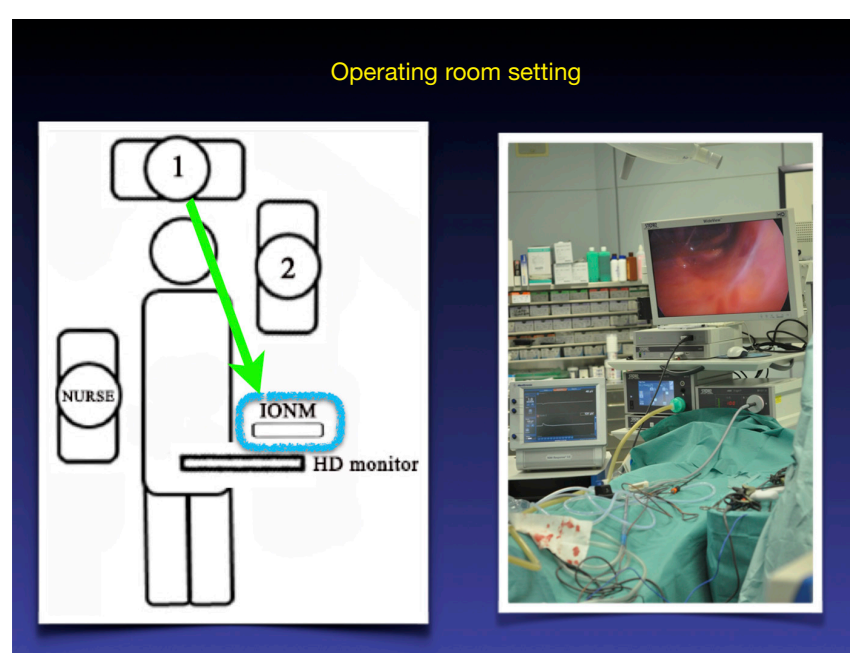

Figure 2 The operative room setting. IONM, intraoperative nerve monitoring; HD, high definition.

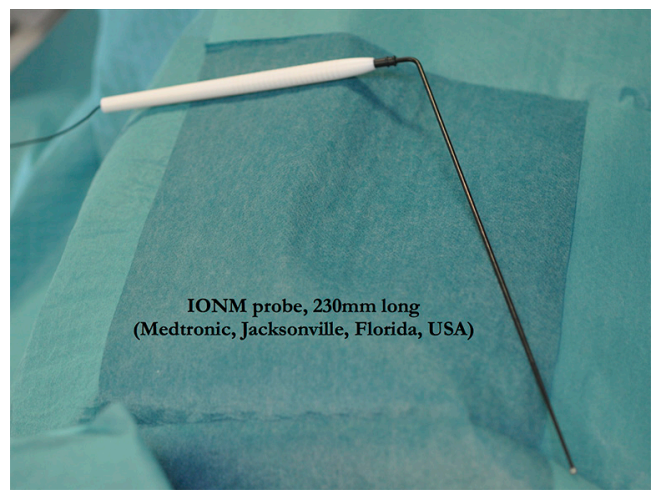

Figure 3 A single use, $230 \mathrm{~mm}$ ball tip long stimulation probe, monopolar (product n.PSP1002, Medtronic, Jacksonville, Florida, USA). The probe is connected with the IONM system. The probe is FDA approved. IONM, intraoperative nerve monitoring.

(I) achievement of vagal nerve (V1, V2), recurrent laryngeal nerve (RLN) (R1, R2) and superior laryngeal nerve (S1, S2) determinations; (II) no additional neck skin incisions; (III) precise contact with nerve structure and thus, use of lower intensity stimulation; (IV) versatility (i.e., bilateral use, both lobes dissection, both laryngeal nerves mapping, any port insertion); (V) atraumatic (i.e., ball tip); (VI) tip is flexible, can be adjusted during procedure to reach narrow areas of dissection; (VII) the long stimulating probe is FDA approved.

Possible limits are: (I) availability of the long probe

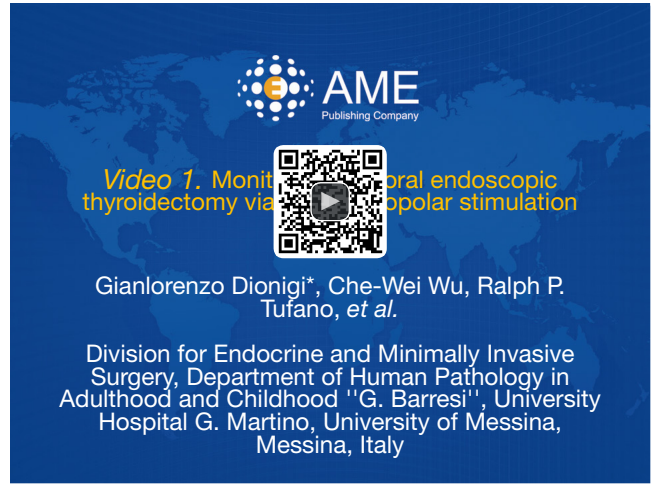

Figure 4 Monitored transoral endoscopic thyroidectomy via long monopolar stimulation probe (25).

Available online: http://asvidett.amegroups.com/article/view/22567

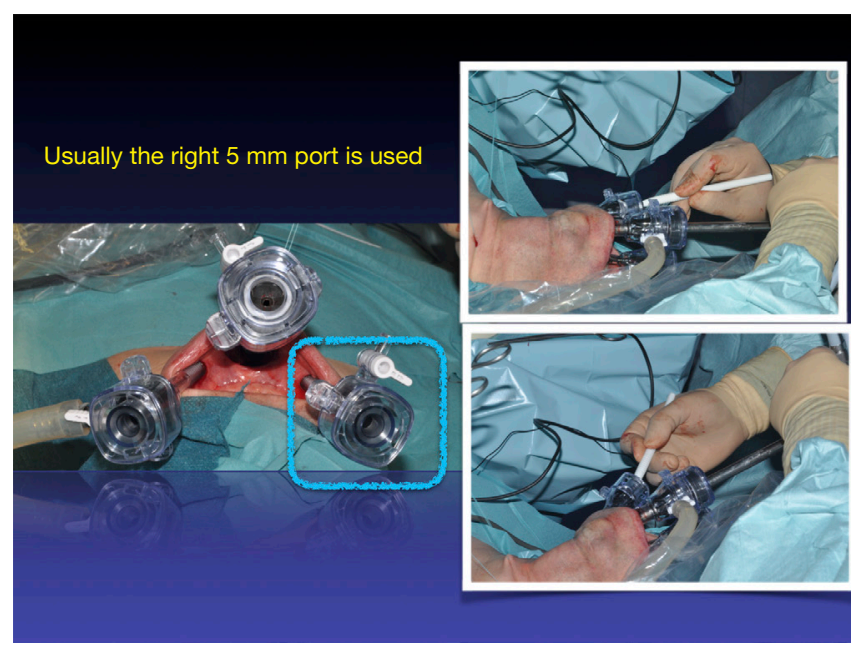

Figure 5 For the right handed surgeon, the long stimulating probe is introduced and used from the right port without any interference with the other endoscopic instruments.

in the operating room and additional cost related; (II) changing instrument; (III) some loss of CO2 insufflation from port; (IV) currently the long stimulating probe is not available in the incrementing probe/remote control mode of application; (V) disposable.

$\mathrm{VN}$ stimulation both V1 and V2 was achieved without carotid sheath dissection by simply and gently applying the stimulating probe on the carotid sheath with $2-3 \mathrm{~mA}$ stimulation intensity.

\section{Role of team members}

Monitoring in TOETVA requires training. Surgeons and 
Anesthesiologist must have accumulated high experience with IONM standards, equipments, troubleshooting algorithms, limits and failures. It is unwise to use IONM for the first time with TOETVA, without any prior nerve monitoring experience.

\section{Postoperative management}

No dressing after TOETVA. Mouthwash 3 times per day are prescribed for 5-7 days. All patients started an oral diet on day 0 postoperatively, evening. Patients are mobilized from bad at +4 hours postoperatively. Patients can take a shower and man shave the same evening of surgery. Patients can sunbathe on the following weekend. Patients may develop mild emphysema, ecchymosis in the neck or swelling of the lower lip: these are treated conservatively and resolve spontaneously within 24-48 hours. A sensory disorder around the chin usually persist for 2-4 weeks after surgery.

Discharge is guided by the common rules of the thyroid surgery, after careful evaluation by surgeon, endocrinological and anesthesiological specialist, serum calcium dosage and after neck, mouth and postoperative laryngeal evaluation. Oral antibiotics and mouthwash 3 times per day are prescribed for 5-7 days. Patient is followup in clinics.

\section{Conclusions}

Monitored TOETVA with the long stimulating probe is feasible and safe. There were no instances of IONM equipment interference with the other endoscopic instruments. The first advantage is that IONM facilitates RLN and SLN identification (i.e., navigation). Second, it enables testing of RLN and SLN function (i.e., monitoring). Third, it enables corrective action at three stages of surgery: (I) during blunt dissection; (II) during use of energy-based devices, and (III) during thyroid gland retraction. Notably, retraction of thyroid gland by grasp forceps or by forceps can cause excessive traction and subsequent functional damage of the RLN. Fourth, it enables evaluation of RLN function by vagus nerve stimulation on one side before proceeding to the contralateral lobe. This advantage is particular important for bilaterally approaches. The fifth advantage is that, for novice surgeons, IONM increases confidence in performing TOETVA procedures. However, skilled surgeons can also use IONM to explore new applications of TOETVA.

\section{Acknowledgements}

None.

\section{Footnote}

Conflicts of Interest: The authors have no conflicts of interest to declare.

Informed Consent: Informed consent was obtained from all individual participants included in the video.

\section{References}

1. Witzel K, von Rahden BH, Kaminski C, et al. Transoral access for endoscopic thyroid resection. Surg Endosc 2008;22:1871-5.

2. Benhidjeb T, Wilhelm T, Harlaar J, et al. Natural orifice surgery on thyroid gland: totally transoral video-assisted thyroidectomy (TOVAT): report of first experimental results of a new surgical method. Surg Endosc 2009;23:1119-20.

3. Wilhelm T, Metzig A. Endoscopic minimally invasive thyroidectomy: first clinical experience. Surg Endosc 2010;24:1757-8.

4. Wilhelm T, Metzig A. Endoscopic minimally invasive thyroidectomy (eMIT): a prospective proof-of-concept study in humans. World J Surg 2011;35:543-51.

5. Anuwong A. Transoral endoscopic thyroidectomy vestibular approach: a series of the first 60 human cases. World J Surg 2016;40:491-7.

6. Liu E, Qadir Khan A, Niu J, et al. Natural orifice total transtracheal endoscopic thyroidectomy surgery: first reported experiment. J Laparoendosc Adv Surg Tech A 2015;25:586-91.

7. Woo SH. Endoscope-assisted transoral thyroidectomy using a frenotomy incision. J Laparoendosc Adv Surg Tech A 2014;24:345-9.

8. Benhidjeb T, Stark M. Endoscopic minimally invasive thyroidectomy (eMIT): safety first! World J Surg 2011;35:1936-7; author reply 1938-9.

9. Clark JH, Kim HY, Richmon JD. Transoral robotic thyroid surgery. Gland Surg 2015;4:429-34.

10. Lee HY, Richmon JD, Walvekar RR, et al. Robotic transoral periosteal thyroidectomy (TOPOT): experience in two cadavers. J Laparoendosc Adv Surg Tech A 2015;25:139-42.

11. Lee HY, You JY, Woo SU, et al. Transoral periosteal 
thyroidectomy: cadaver to human. Surg Endosc 2015;29:898-904.

12. Lee HY, Hwang SB, Ahn KM,et al. The safety of transoral periosteal thyroidectomy: results of Swine models. J Laparoendosc Adv Surg Tech A 2014;24:312-7.

13. Inabnet WB 3rd, Suh H, Fernandez-Ranvier G. Transoral endoscopic thyroidectomy vestibular approach with intraoperative nerve monitoring. Surg Endosc 2017;31:3030.

14. Park JO, Kim MR, Kim DH, et al. Transoral endoscopic thyroidectomy via the trivestibular route. Ann Surg Treat Res 2016;91:269-72.

15. Witzel K, Hellinger A, Kaminski C, et al. Endoscopic thyroidectomy: the transoral approach. Gland Surg 2016;5:336-41.

16. Udelsman R, Anuwong A, Oprea AD, et al. Trans-oral vestibular endocrine surgery: a new technique in the United States. Ann Surg 2016;264:e13-6.

17. Dralle H. Impact of modern technologies on quality of thyroid surgery. Langenbecks Arch Surg 2006;391:1-3.

18. Dionigi G, Lavazza M, Bacuzzi A, et al. Transoral endoscopic thyroidectomy vestibular approach (TOETVA): From A to Z. Surg Technol Int 2017;30:103-12.

19. Dionigi G, Bacuzzi A, Lavazza M, et al. Transoral

doi: 10.21037 /jovs.2017.12.25

Cite this article as: Dionigi $\mathrm{G}, \mathrm{Wu} \mathrm{CW}$, Tufano RP, Rizzo AG, Anuwong A, Sun H, Carcoforo P, Antonino C, Portinari M, Kim HY. Monitored transoral endoscopic thyroidectomy via long monopolar stimulation probe. J Vis Surg 2018;4:24. endoscopic thyroidectomy via vestibular approach: operative steps and video. Gland Surg 2016;5:625-7.

20. Dionigi G, Tufano RP, Russell J, et al. Transoral thyroidectomy: advantages and limitations. J Endocrinol Invest 2017;40:1259-63.

21. Dionigi G, Bacuzzi A, Lavazza M, et al. Transoral endoscopic thyroidectomy: preliminary experience in Italy. Updates Surg 2017;69:225-34.

22. Dionigi G, Rovera F, Boni L. Commentary on transoral access for endoscopic thyroid resection : Witzel K, von Rahden BH, Kaminski C, Stein HJ (2008) Transoral access for endoscopic thyroid resection. Surg Endosc 22(8):18711875. Surg Endosc 2009;23:454-5; discussion 456.

23. Randolph GW, Dralle H, International Intraoperative Monitoring Study Group, et al. Electrophysiologic recurrent laryngeal nerve monitoring during thyroid and parathyroid surgery: international standards guideline statement. Laryngoscope 2011;121:S1-16.

24. Dionigi G, Boni L, Rovera F,et al. Neuromonitoring and video-assisted thyroidectomy: a prospective, randomized case-control evaluation. Surg Endosc 2009;23:996-1003.

25. Dionigi G, Wu CW, Tufano RP, et al. Monitored transoral endoscopic thyroidectomy via long monopolar stimulation probe. Asvide 2018;5:044. Available online: http://asvidett. amegroups.com/article/view/22567 